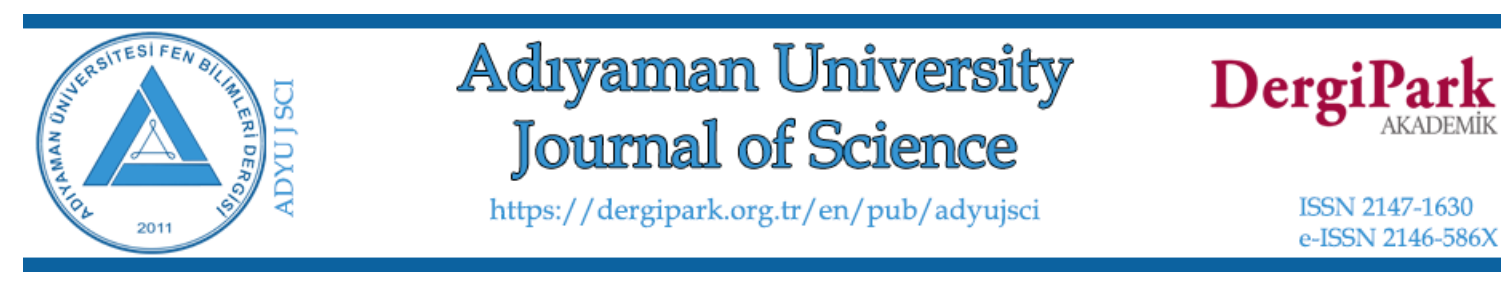

\title{
Investigation of the DKP Equation for A Two-Dimensional Black Hole
}

\author{
Evrim Ersin KANGAL ${ }^{1, *}$, Ali HAVARE ${ }^{2}$ \\ ${ }^{1}$ Mersin University, School of Applied Technology and Management of Erdemli, Department of Computer \\ Technology and Information Systems, 33740, Mersin, Turkey \\ evrimersin@gmail.com,ORCID:0000-0001-5906-3143 \\ ${ }^{2}$ Mersin University, Faculty of Arts and Sciences, Department of Physics, 33343, Mersin, Turkey \\ ahavare@gmail.com, ORCID: 0000-0002-6476-7708
}

\begin{abstract}
In the present study, we firstly investigated the spatial properties of event horizon of a twodimensional black hole. Then we solve Duffin-Kemmer-Petiau (DKP) equation for such a black hole metric depending on the signs of spatial variable. After obtaining the exact solutions, we determine thermal parameters related to this metric. Finally, the harmonic oscillation behavior of the system is evaluated.
\end{abstract}

Keywords: DKP equation; Black hole; Thermal parameters; Harmonic oscillation.

\section{İki Boyutlu Bir Karadelik için DKP Denkleminin Araştırılması}

\section{Öz}

$\mathrm{Bu}$ çalışmada ilk olarak iki boyutlu bir kara deliğin olay ufkunun uzaysal özellikleri incelendi. Daha sonra böyle bir karadelik metriği için uzaysal değişkenin işaretine bağlı olarak DKP denklemi çözüldü. Tam çözümler elde edildikten sonra bu metrik ile ilişkili isıl nicelikler belirlendi. Son olarak sistemin harmonik salınım davranışı değerlendirildi.

Anahtar Kelimeler: DKP Denklemi; Kara delik; Isıl parametreler; Harmonik osilasyon. 


\section{Introduction}

General relativity is one of the most attractive topics in modern theoretical physics [1-3]. In 1915, Einstein proposed that the gravity is a result of geometry and formulated it with the following equation,

$$
G_{\mu \nu}=8 \pi G T_{\mu \nu}
$$

where $T_{\mu \nu}$ is the stress-energy tensor of matter fields, $G$ is the Newtonian constant of gravitation and $G_{\mu \nu}$ is the Einstein tensor given by

$$
G_{\mu \nu}=R_{\mu \nu}-\frac{1}{2} g_{\mu \nu} R
$$

$R_{\mu \nu}$ is the Ricci curvature tensor, $g_{\mu \nu}$ is the metric tensor, and $R$ is the scalar curvature. Black hole is generally evaluated as a new mathematical or physical mechanism used to construct a link between gravity and quantum theory [4-5]. However, the dynamics of physical properties of a black hole existing in a four-dimensional curved spacetime can be sometimes extremely hard to understand thanks to some difficulties in quantizing gravity. For this reason, one of the best ways of overcoming these challenges is to reduce to a two-dimensional spacetime [6-7]. In such a spacetime, the Einstein field equation reduces to,

$$
R-\Lambda=8 \pi G T
$$

where $T$ is the trace of two-dimensional energy momentum tensor and $\Lambda$ is cosmological constant. Mann proposed to employ the following line element for a black hole by solving the above Einstein field equation in two dimensional spacetime [8]

$$
d s^{2}=-\alpha(x) d t^{2}+\frac{1}{\alpha(x)} d x^{2}
$$

where

$$
\alpha(x)=-\frac{\Lambda}{2} x^{2}+2 M|x|-C
$$

$C$ is an arbitrary constant and $M$ represents a positive energy source. Here, the number of distinct classes of solutions is dependent on the signs of $\Lambda, C$, and $M$. For $\Lambda=0$, the location of event horizon is [8]

$$
|x|=\frac{C}{2 M}
$$


According to this equation, the existence of an event horizon appears if $C$ and $M$ are of the same sign. If Eqn. (6) is plotted for $\Lambda=0$ [8], we obtain
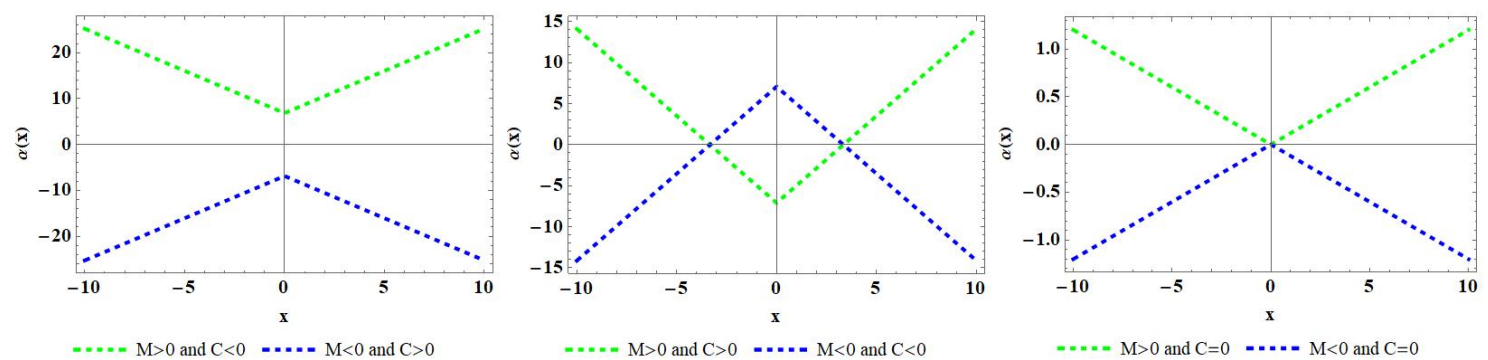

$=-=-\mathrm{M}>0$ and $\mathrm{C}=0 \quad \ldots . .-\mathrm{M}<0$ and $\mathrm{C}=0$

Figure 1: Dependence of $\boldsymbol{\alpha}(\boldsymbol{x})$ scale factor for setting $\boldsymbol{\Lambda}=\mathbf{0}$ and different values of $M$ and $C$

As shown in Fig. 1, the horizon is surrounded by a timelike region for positive $\boldsymbol{M}$ while it is enclosed by a spacelike region for negative $\boldsymbol{M}$ at $\boldsymbol{C}=\mathbf{0}$ case or right-side graph. In addition, we see two distinct horizons for $\boldsymbol{C} \neq \mathbf{0}$ case or center-side graph. Finally, any horizon has not been observed for left-side graph since the absolute value of $\boldsymbol{x}$ is greater than zero.

If $\boldsymbol{\Lambda} \neq \mathbf{0}$, event horizon is placed at [8]

$$
|x|=\frac{2 M \pm \sqrt{4 M^{2}-2 C \Lambda}}{\Lambda}
$$

From the above equation, one can say that the existence of an event horizon is mainly in accordance with the $\boldsymbol{C} \boldsymbol{\Lambda} \leq \mathbf{2} \boldsymbol{M}^{\mathbf{2}}$ equality. If we make a plot of Eqn. (7) under this circumstance [8], we get

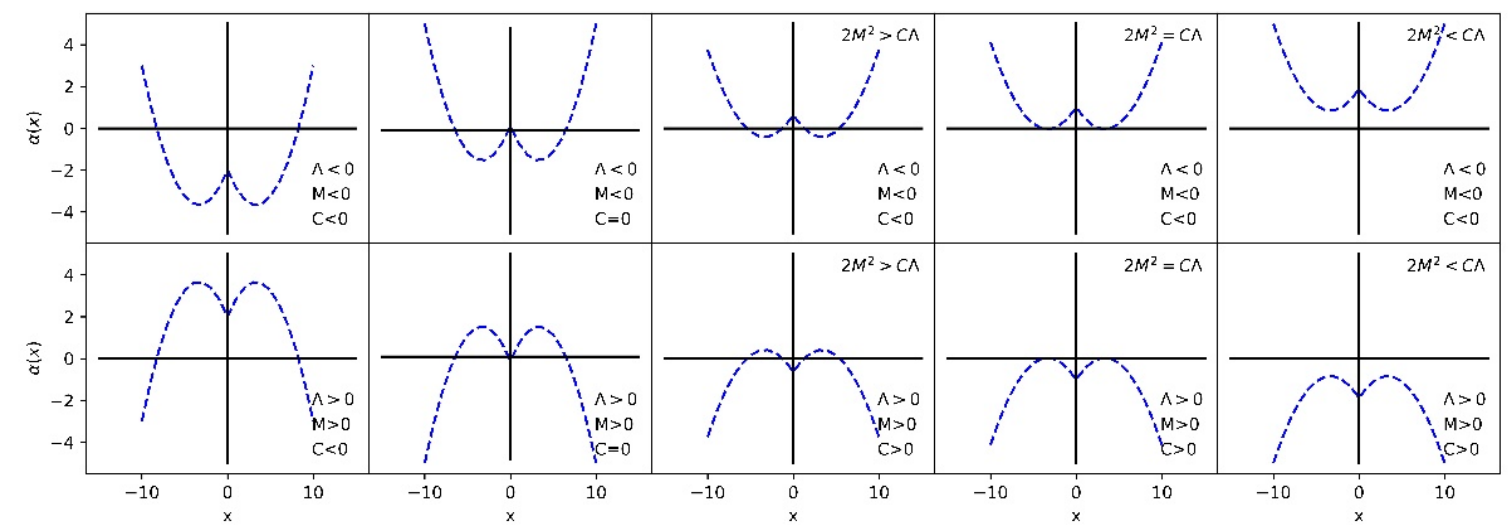

Figure 2: Type of solutions for running values of $\boldsymbol{\Lambda}$

From Fig. 2, we can easily say that $\alpha(x)$ equation have ten distinct of solutions whose behaviour are anti-symmetric structure with respect to each other. Further, any exist of restriction does not appear in the first two columns while the presence of a restriction exists in the other columns. 
The DKP equation was proposed by Duffin, Kemmer and Petiau [9-11] and is mathematically identical to the general form of Dirac equation but it is uniquely characterized by $\boldsymbol{\beta}$ matrices instead of $\boldsymbol{\gamma}$ matrices. However, we sometimes encounter some difficulties to solve the DKP equation in $(3+1)$ dimensions since it is a 16 -component wave equation. An alternative way to overcome this problem is to evaluate the DKP equation in the lower dimensions in order to obtain the behavior of semi-classical and quantum gravity. In the literature, we find many studies related to the general relativity in $(1+1)$-dimensions [12-15].

In this work, we firstly obtained the exact solution of the DKP equation for the line element of a two-dimensional black hole described at Eqn. (4). In the second section, we investigate thermal parameters related to these solutions. Then the harmonic oscillation frequency is obtained in section 4. Last section is devoted to discussing our results.

\section{The Exact Solution of the DKP Equation in (1+1) - Dimensions}

The DKP equation in curved spacetime is given by [16-18]

$$
\left[i \beta^{\mu}\left(\partial_{\mu}-\Sigma_{\mu}\right)-m\right] \Psi_{K}(t, x)=0
$$

where $m$ is particle mass, $\beta^{\mu}=\gamma^{\mu} \otimes I+I \otimes \gamma^{\mu}$ are the Kemmer matrices, $\gamma^{\mu}=e_{(i)}^{\mu} \gamma^{(i)}$, $\Psi_{K}(t, x)$ shows the 16-component Kemmer wave function and $\Sigma_{\mu}$ are generally known as the spinorial connections defined by

$$
\Sigma_{\mu}=\Gamma_{\mu} \otimes I+I \otimes \Gamma_{\mu}
$$

where $I$ is the $4 \otimes 4$ identity matrices and $\Gamma_{\mu}$ are the spin connections for spin-1/2 particle calculated with the following equation

$$
\Gamma_{\lambda}=-\frac{1}{8} g_{\mu \alpha} \Gamma_{v \lambda}^{\alpha}\left[\gamma^{\mu}, \gamma^{\nu}\right]
$$

and $\Gamma_{\nu \lambda}^{\alpha}$ are the Christoffel symbols that are directly written in terms of the metric tensor as

$$
\Gamma_{\mu \nu}^{\alpha}=\frac{1}{2} g^{\alpha \beta}\left(\partial_{\mu} g_{\beta \nu}+\partial_{\nu} g_{\beta \mu}-\partial_{\beta} g_{\mu \nu}\right)
$$

Unal [19-21] proved that the Kemmer wave function does not change under local Lorentz transformations, when the DKP particles are mathematically considered as a system of the product of two-identical spin-1/2 particles. Hence, the Kemmer wave function and $\beta^{\mu}$ matrices are reduced to 


$$
\begin{aligned}
& \Psi_{K}(t, x)=\Psi_{D}(t, x) \otimes \Psi_{D}(t, x)=\left[\begin{array}{l}
\rho \\
\varphi
\end{array}\right] \otimes\left[\begin{array}{l}
\rho \\
\varphi
\end{array}\right]=\left[\begin{array}{l}
\rho \rho \\
\rho \varphi \\
\rho \varphi \\
\varphi \varphi
\end{array}\right]=\left[\begin{array}{l}
h_{1} \\
h_{0} \\
h_{0} \\
h_{2}
\end{array}\right] e^{-i w t} \\
& \beta^{\mu}(x)=\sigma^{\mu}(x) \otimes I+I \otimes \sigma^{\mu}(x)
\end{aligned}
$$

since the usual Dirac matrices are replaced with the Pauli spin matrices, and also the Kemmer wave function oscillates freely with time. If we choose the Pauli matrices as $\sigma^{\mu}=$ $\left(\sigma^{3}=\left(\begin{array}{cc}1 & 0 \\ 0 & -1\end{array}\right),-i \sigma^{2}=\left(\begin{array}{cc}0 & -1 \\ 1 & 0\end{array}\right)\right)$ and substitute Eqn. (12) and Eqn. (13) into Eqn. (8), we get the following set of coupled differential equations:

$$
\begin{aligned}
& \left(2 i w \alpha^{-\frac{1}{2}}-m\right) h_{1}+2\left(\alpha^{\frac{1}{2}} \partial_{x}-\frac{\alpha^{\prime}}{2 \alpha^{\frac{1}{2}}}\right) h_{0}=0 \\
& \left(2 i w \alpha^{-\frac{1}{2}}+m\right) h_{2}+2\left(\alpha^{\frac{1}{2}} \partial_{x}-\frac{\alpha^{\prime}}{2 \alpha^{\frac{1}{2}}}\right) h_{0}=0 \\
& h_{0}=\frac{\alpha^{\frac{1}{2}}}{m} \partial_{x}\left(h_{2}-h_{1}\right)
\end{aligned}
$$

After some mathematical algebra, the above equations yields

$$
\begin{aligned}
& h_{1}+h_{2}=2 i w \alpha^{-\frac{1}{2}}\left(h_{2}+h_{1}\right) \\
& \left(\frac{\partial^{2}}{\partial x^{2}}+\frac{w^{2}}{\alpha^{2}}+\frac{m^{2}}{4} \frac{1}{\alpha}\right) \Phi=0
\end{aligned}
$$

where $\Phi=h_{2}-h_{1}$. If we insert Eqn. (5) into Eqn. (16), we find the following equation

$$
\left(\frac{\partial^{2}}{\partial x^{2}}+\frac{4 w^{2}}{\left(\Lambda x^{2}-4 M|x|+2 C\right)^{2}}-\frac{m^{2}}{2} \frac{1}{\Lambda x^{2}-4 M|x|+2 C}\right) \Phi(x)=0
$$

The exact solution of Eqn. (17) depends on the sign of the $x$ variable:

- $\boldsymbol{x}<\mathbf{0}$ case: Eqn. (17) becomes

$$
\left(\frac{\partial^{2}}{\partial x^{2}}+\frac{4 w^{2}}{\left(\Lambda x^{2}+4 M x+2 C\right)^{2}}-\frac{m^{2}}{2} \frac{1}{\Lambda x^{2}+4 M x+2 C}\right) \Phi(x)=0
$$

This second order differential equation resembles the associated Legendre differential equation, and solutions are obtained as follows [22] 


$$
\Phi(x)=\sqrt{\Lambda x^{2}+4 M x+2 C}\left[C_{1} P_{n}^{m}\left(\frac{x+\frac{M}{\Lambda}}{\sqrt{\left(\frac{M}{\Lambda}\right)^{2}-\frac{2 C}{\Lambda}}}\right)+C_{2} Q_{n}^{m}\left(\frac{x+\frac{M}{\Lambda}}{\sqrt{\left(\frac{M}{\Lambda}\right)^{2}-\frac{2 C}{\Lambda}}}\right)\right]
$$

- $\boldsymbol{x}>\mathbf{0}$ case: Eqn. (17) takes

$$
\left(\frac{\partial^{2}}{\partial x^{2}}+\frac{4 w^{2}}{\left(\Lambda x^{2}-4 M x+2 C\right)^{2}}+\frac{m^{2}}{2} \frac{1}{\Lambda x^{2}-4 M x+2 C}\right) \Phi(x)=0
$$

Similarly, for the solution of this equation, we find the following result [22]

$$
\Phi(x)=\sqrt{\Lambda x^{2}-4 M x+2 C}\left[C_{1} P_{n}^{m}\left(\frac{x-\frac{M}{\Lambda}}{\sqrt{\left(\frac{M}{\Lambda}\right)^{2}-\frac{2 C}{\Lambda}}}\right)+C_{2} Q_{n}^{m}\left(\frac{x-\frac{M}{\Lambda}}{\sqrt{\left(\frac{M}{\Lambda}\right)^{2}-\frac{2 C}{\Lambda}}}\right)\right]
$$

where

$$
\begin{aligned}
& n=\frac{1}{2}\left(-1+\sqrt{1+2 m^{2} \Lambda}\right) \\
& m=\sqrt{1+\frac{\left(\frac{2 w}{\Lambda}\right)^{2}}{-\left(\frac{M}{\Lambda}\right)^{2}+\frac{2 C}{\Lambda}}}
\end{aligned}
$$

The values of $\mathrm{m}$ parameter are integers because of definition. Thus, the energy is obtained as

$$
E_{l}=\hbar \sqrt{2 M^{2}-C \Lambda l}
$$

where $l$ is a positive integer number.

\section{Calculation of the Thermal Quantities}

The partition function of the DKP particle is given by [23]

$$
Z(\beta)=\sum_{l=0}^{\infty} e^{-\beta\left(E_{l}-E_{0}\right)}=\sum_{l=0}^{\infty} e^{-\hbar \beta \sqrt{2 M^{2}-C \Lambda} l}
$$

Before determining the thermal quantities, we need to test the convergence of series of Eqn. (25) by using the integral test which shows whether the integral converges or diverges. The function from Eqn. (25) is

$$
f(x)=e^{-\hbar \beta \sqrt{2 M^{2}-C \Lambda} l}
$$

where $\hbar=1$ is adopted. If the integral test is performed to Eqn. (26), we obtain

$$
\int_{0}^{\infty} f(l) d l=\frac{1}{\sqrt{2 M^{2}-C \Lambda}} \frac{1}{\beta}
$$


so, function is convergent. In the meantime, the numerical partition function is calculated with the help of a method depending on the Euler-MacLaurin formula [24] defined by the following equation

$$
\sum_{l=0}^{\infty} f(x)=\frac{1}{2} f(0)+\int_{0}^{\infty} f(x) d x-\sum_{p=1}^{\infty} \frac{B_{2 p}}{(2 p) !} f^{2 p-1}(0)
$$

where $B_{2 p}$ are the Bernoulli numbers, $f^{2 p-1}$ is the derivative of order $2 p-1$. If we write the exact form of partition function $Z$ by using Eqn. (28), we get

$$
Z(\beta)=\frac{1}{2}+\frac{1}{\sqrt{2 M^{2}-C \Lambda}} \frac{1}{\beta}+\frac{\sqrt{2 M^{2}-C \Lambda}}{12} \beta-\frac{\left(\sqrt{2 M^{2}-C \Lambda}\right)^{3}}{720} \beta^{3}
$$

where the terms up to $p=2$, with $B_{2}=\frac{1}{6}$, and $B_{6}=-\frac{1}{30}$ are chosen. If the following thermal quantities like the Helmholtz free energy, the mean energy, the entropy, and the specific heat are calculated by using the partition function,

$$
\begin{aligned}
& F=-\frac{1}{\beta} \ln Z \\
& S=\beta^{2} \frac{\partial F}{\partial \beta} \\
& <E>=-\frac{\partial \ln Z}{\partial \beta} \\
& C_{v}=-\beta^{2} \frac{\partial<E>}{\partial \beta}
\end{aligned}
$$

the resulting solutions are messy, so we will discuss these quantities numerically with the help of MATHEMATICA [25] and thermal quantities given by Eqn. (30-33) are plotted depending on some values of $C$ parameter for $M \neq 0$ and $\Lambda \neq 0$ by considering $C \Lambda \leq 2 M^{2}$ restriction and are shown in Fig. 3. From Fig. 3, we can say that thermal capacity is going to a fixed value at high temperature since a physical system has always a constant thermal capacity and also, we observe the same behavior in the entropy. Besides, the behaviour of mean energy is reasonable because the contribution coming from every dimension is almost $\frac{k T}{2}$, and then total energy is approximately equal to $k T$ like graph. 

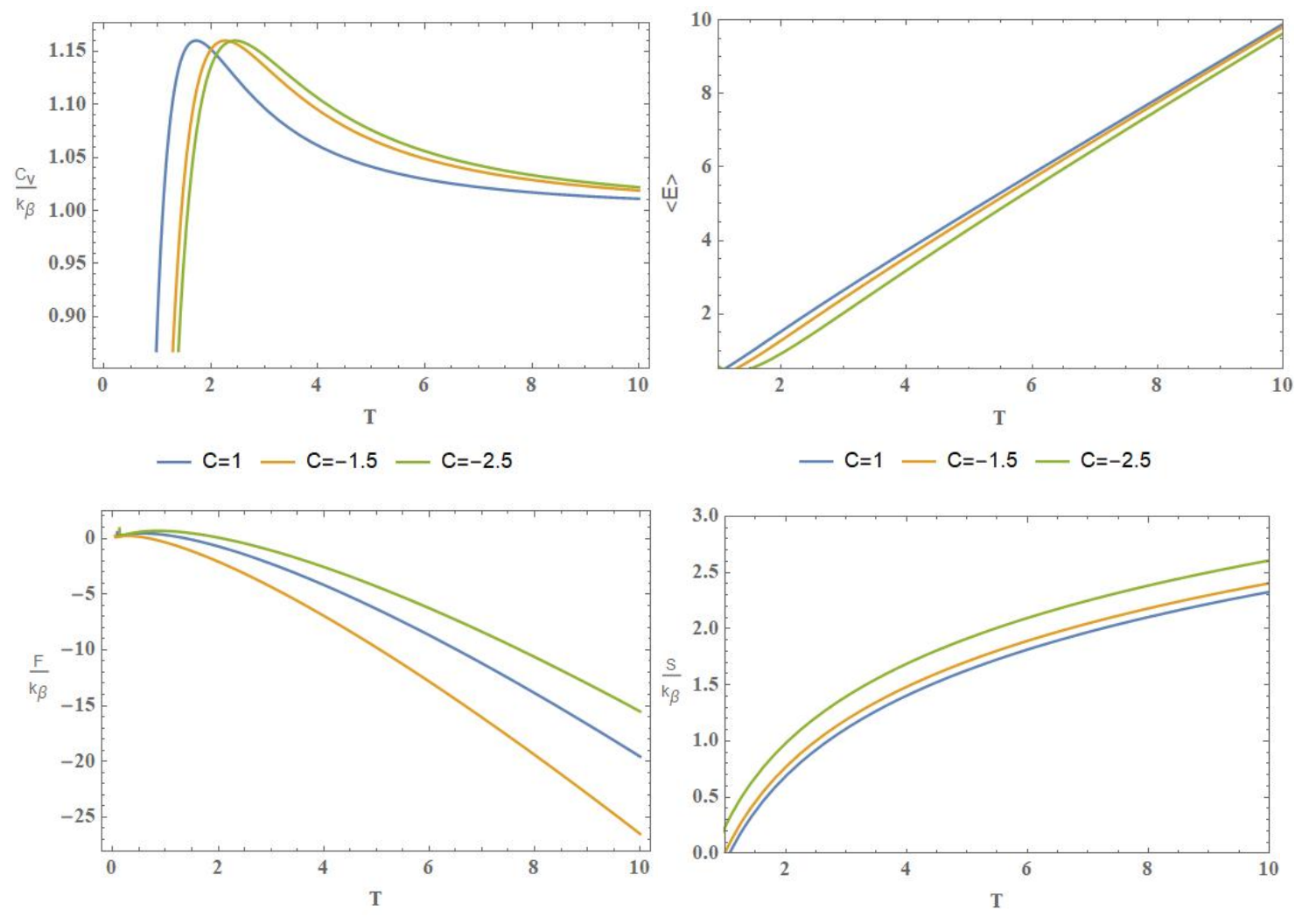

$-C=1-C=-1.5-C=-2.5$

$-\mathrm{C}=1-\mathrm{C}=-1.5-\mathrm{C}=-2.5$

Figure 3: Thermal quantities depending on $C$ for $M \neq 0$ and $\Lambda \neq 0$

\section{The Harmonic Oscillator Behavior of the DKP Particles}

To determine the range of oscillations of DKP particles, the form of the quadratic differential equation must be transformed to resemble the form of the differential equation of the harmonic oscillator given by

$$
\left[\frac{\partial^{2}}{\partial x^{2}}+w^{2}\right] y(x)=0
$$

where w represents the behavior of harmonic oscillation frequency. If we compare Eqn. (34) with Eqn. (17), we get

$$
w^{2}=\frac{4 w^{2}}{\left(\Lambda x^{2}-4 M|x|+2 C\right)^{2}}-\frac{m^{2}}{2} \frac{1}{\Lambda x^{2}-4 M|x|+2 C}
$$

and the following inequality corresponds to the range of above equation:

$$
\frac{2 M}{\Lambda}-\sqrt{\left(\frac{M}{\Lambda}\right)^{2}+\left(\frac{2 w}{\Lambda}\right)^{2}-\frac{2 C}{\Lambda}}<|x|<\frac{2 M}{\Lambda}+\sqrt{\left(\frac{M}{\Lambda}\right)^{2}+\left(\frac{2 w}{\Lambda}\right)^{2}-\frac{2 C}{\Lambda}} .
$$




\section{Conclusions}

In this study, we solved the DKP equation for a two-dimensional black hole. In this case, the general solution is obtained by means of the associated Legendre Polynomials based on the signs of the $x$ variable. Energy spectrum was obtained with the help of m parameter of Associated Legendre polynomials, and thermal quantities were calculated from energy spectrum. When the thermal quantities are graphically examined depending on the temperature, it is seen that the increases in the temperature alter these quantities. In addition, these changes show shifts depending on the different values of the $C$ parameter of the metric. After all, we obtained the harmonic behavior of the DKP particles by determining the oscillation range. The oscillator behavior of particle is restricted in a spatial regime given by Eqn. (36). In conclusion, we can say that studying in the lower dimensions provides insights into the higher dimensions.

\section{References}

[1] Koyama, K., Gravity beyond general relativity, International Journal of Modern Physics D, 27 (15), 1848001, 2018. 1954.

[2] Gupta, S.N., Gravitation and electromagnetism, Physical Review, 96(6), 1683-1685,

[3] Boulware, D.G., Deser, S., Classical general relativity derived from quantum gravity, Annals of Physics, 89(1), 193-240, 1975.

[4] Hawking, S.W., Black holes in general relativity, Communications in Mathematical Physics, 25(2), 152-166, 1972. 1976.

[5] Hawking, S.W., Black holes and thermodynamics, Physical Review D, 13(2), 191-197,

[6] Teitleboim, C., Gravitation and Hamiltonian structure in two spacetime dimensions, Physics Letters B, 126(2), 41-45, 1983.

[7] Jackiw, R., Lower Dimensional Gravity, Nuclear Physics B, 252, 343-356, 1985.

[8] Mann, R.B., Shiekh, A., Tarasov, L., Classical and quantum properties of towdimensional black holes, Nuclear Physics B, 341(1),134-154, 1989.

[9] Duffin, R.J., On the characteristic matrices of covariant systems, Physical Review, 54, $1114,1938$.

[10] Kemmer, N., The particle aspect of meson theory, Proceeding of the Royal Society A, 173, 91-116, 1939.

[11] Petiau, G., PhD thesis, Academie Royale de Belgique, Classe des Sciences, Memoires, Collection 8, 1936.

[12] Lunardi, J.T., A note on the Duffin-Kemmer-Petiau equation in $(1+1)$ space-time dimensions, Journal of Mathematical Physics, 58, 123501-123505, 2017.

[13] Lunardi, J.T., Pimentel, B.M., Teixeiri, R.G., Valverde, J.S., Remarks on DuffinKemmer-Petiau theory and gauge invariance, Physics Letter A, 268(3), 165-173, 2000. 
[14] Sogut, K., Havare, A., Transmission resonances in the Duffin-Kemmer-Petiau equation in $(1+1)$ dimensions for an asymmetric cusp potential, Physica Scripta, 82(4), 045013, 2010.

[15] Parker, L., Toms, D.J., Quantum Field Theory in curved spacetime, Cambridge University Press, 2009.

[16] Merad, M., DKP equation with smooth potential and position-dependent mass, International Journal of Theoretical Physics, 46, 2105-2118, 2007.

[17] Cheraitia, B.B., Boudjedaa, T., Solution of DKP equation in Woods-Saxon potential, Physics Letter A, 338(2), 97-107, 2005.

[18] Yasuk, F., Berkdemir, A., Onem, C., Exact Solutions of the Duffin-Kemmer-Petiau Equation for the Deformed Hulthen Potential, Physica Scripta, 71(4), 340-343, 2005.

[19] Unal, N., Duffin-Kemmer-Petiau equation, Proca equation and Maxwells equation in (1+1) D, Concepts of Physics, 2, 273, 2005.

[20] Unal, N., Path integral quantization of a spinning particle, Foundations of Physics, 28, 755-762, 1998.

[21] Unal, N., A simple model of the classical Zitterbewegung: Photon wave function, Foundations of Physics, 27, 731-746, 1997.

[22] Abramowitz, M., Stegun, I.A., Handbook of mathematical functions, National Bureau of Standards Applied Mathematics, 55, 1964.

[23] Boumali, A., One-dimensional thermal properties of the Kemmer oscillator, Physica Scripta, 76(6), 669-673, 2007.

[24] Pacheco, M.H., Landim, R.R., Almeida, C.A.S., One-dimensional Dirac oscillator in a thermal bath, Physics Letters A, 311, 93-96, 2003.

[25] Wolfram Research Company, Mathematica 9.0, 2012. 